\title{
Conceptualizing Knowledge in Digital Innovation Labs
}

\author{
Satu Iho \\ University of Lausanne \\ satu.iho@unil.ch
}

\author{
Stéphanie Missonier \\ University of Lausanne \\ stephanie.missonier@unil.ch
}

\begin{abstract}
This paper examines the types of knowledge involved in IT exploration and exploitation; and how individuals can manage them. We focus on a particular organizational context described in previous research where individuals transfer between a digital innovation lab (DIL) and the existing organization for periods of time. Drawing on existent literature, we conceptualize six types of knowledge and relate them to the behaviors of learning, applying and intentional forgetting. We illustrate our conceptualization with two vignettes based on empirical data. Our conceptualization raises awareness of potential knowledge-related challenges associated with DILs, and provides insight on the composition of knowledge managed in a DIL to support fruitful IT exploration and digital innovation. Given the importance of digital innovation for today's organizations, understanding the types of knowledge in a DIL set-up is of vital importance.
\end{abstract}

\section{Introduction}

Knowledge and learning are seen as fundamental building blocks for an organization's digital innovation capability [1]. In particular, combining knowledge from an organization's mature existing knowledge base with new, external knowledge has the potential for radical innovations that are hard for competitors to imitate [2]. In examining this capability, scholars often leverage the concepts of exploration and exploitation. Foundational to the organizational learning framework by March [3], exploration involves the "pursuit of new knowledge", while exploitation is characterised by the "use and development of things already known" [4 p. 105]. Put differently, exploration consciously moves away from the existing knowledge base and organizational routines, while exploitation leverages an organization's existing knowledge base [5]. Applying these concepts to an IT context, IT exploration consists of searching for and choosing new technologies and methodologies that can be used for value creation through digital innovation. In contrast, IT exploitation focuses on maintaining and incrementally improving existing systems and methodologies to add continued value to the organization [6].

As the two activities of exploration and exploitation are fundamentally different, separating them into differentiated organisational units has been suggested as an approach to simultaneously pursue both [7]. Accordingly, many incumbent organizations are setting up separate organizational units referred to as digital innovation labs (DILs) which are dedicated to IT exploration, whilst the existing organization and IT function remain dedicated to IT exploitation. This allows both organizational units-the DIL and existing organization-to deploy their own people, structure, processes and ways of working, and foster a culture that is appropriate for their respective objectives and activities [6]. Such structural separation has been suggested to relieve the tensions arising from the differing characteristics and objectives of the two sets of activities [3, 8]. However, integration between the separated units is also required to allow for sharing of resources and capabilities [9]. This is of particular importance in the context of digital innovation to ensure that any new digital solutions-where digital technologies have been incorporated to products, processes or business models-can provide true business value [10].

In a recent study Holotiuk and Beimborn [10] examine nine DILs of incumbent organizations in distinct industries. The authors find that one way to share knowledge and capabilities is to temporarily transfer individual employees from the existing organization into the DIL for a project and back into the existing organization when the project comes to an end. The purpose is twofold: first, to equip project teams within the DIL with operational knowledge during a project, and second, to help integrate the newly created solution into the organization when a project comes to an end. The transfer therefore allows for the sharing of knowledge between the DIL and the existing organization. This finding provides initial insight into resource and capability sharing between DILs and the rest of the organization. Specifically, it demonstrates how existing and newly created 
knowledge can be shared between the DIL and the organization. However, as Holotiuk and Beimborn also point out, the study gives us little understanding of the types of knowledge being shared and thus involved when IT exploration is separated from the rest of the organization. Our aim with this conceptual paper is to start filling this gap. Given the importance of digital innovation for today's organizations, having detailed understanding of the types of knowledge that contribute to fruitful IT exploration in DILs is of vital importance [11]. We thus pose the following research question: what types of knowledge are involved in IT exploration and exploitation and how can individuals transferring between the DIL and existing organization manage them?

The underlying basis of our conceptualization is knowledge at an individual level. Namely, we base ourselves in the scenario described by Holotiuk and Beimborn [10] of individuals transferring between the DIL and the existing organization. Our focal point is an individual employee that is normally based in an operational role in the existing organization, characterized by exploitation, but that is transferred to the DIL for a period of time for a digital innovation project, characterized by exploration. Work in DILs is however typically done in project teams and DILs are set in an internal organizational environment. We account for this multi-level context by drawing on existing literature from individual, team and organization levels. Moreover, to account for the multifaceted nature of knowledge, we base our conceptualization on existing literature from both organizational science and IS. This holistic approach allows us to conceptualize six types of knowledge involved in exploration activites in DILs. Furthermore, we associate these knowledge types to behaviors required during and after a digital innovation project is carried out in the DIL, i.e. learning, applying and intentionally forgetting.

Our conceptualization makes two key contributions. First, we contribute to practice by delineating the types of knowledge prominent in a DIL context. This can aid managers and organizations setting up DILs to better understand and manage potential knowledge-related challenges individuals transferred into the DIL may encounter. In particular, we draw attention to the challenges temporarily transferred individuals may encounter when required to intentionally forget the exploitation-oriented ways of working that they have grown accustomed to in the existing organization. While this type of knowledge has been empirically observed by previous IS scholars [10], it has not yet been clearly structured and tied into the concepts of exploration and exploitation. Second, our conceptualization provides an initial stepping stone for understanding the composition of knowledge that needs to be managed in a DIL to support fruitful IT exploration and digital innovation [10]. Given the prominence of DILs in incumbent organizations, it is of vital importance to have clarity around the types of knowledge that are most prominent in this organizational set-up.

The rest of this paper is organized as follows. In the next section we review central themes from existing literature that we base our conceptualization on. We do this by levels of analysis, starting with an examination of knowledge at individual level, followed by team and organizational levels. Then, we develop our conceptualization together with its core propositions and illustrate it with the help of two vignettes. These vignettes are based on nine expert interviews with individuals with experience of working in DILs. We conclude by briefly describing the implications of our conceptualization and avenues for future research.

\section{Background}

\subsection{Knowledge as an individual's state of mind}

In their extensive review of the conceptual foundations of knowledge, Alavi and Leidner [12] suggest that knowledge can be viewed as personalized information possessed by an individual. They suggest that knowledge takes a three-tier hierarchical form where data, consisting of facts and raw numbers, is processed and interpreted into information, which, in turn, is converted into knowledge as it is processed and personalized in the mind of an individual. Information becomes knowledge as it is associated with the individual's experiences and contexts in which it is acquired, be they formal or informal. Putting this into an organizational context, the knowledge-based view of the firm posits that a firm's raison d'être is to bring together individuals with specific knowledge and provide them with an environment where they can apply that knowledge in a productive manner [13].

Scholars typically make a distinction between explicit and tacit knowledge. For instance, Grant [13] describes explicit knowledge as knowing about facts and theories, and tacit knowledge as knowing how. Brown and Duguid [14] make a similar distinction when they describe know-what and know-how, where the former refers to knowledge about a topic and the latter allows an individual to apply this knowledge correctly in distinct contexts. As they put it: "knowhow embraces the ability to put know-what into practice" which in turn allows for "knowledge to be made actionable and operational" (p. 95). In this way, explicit and tacit knowledge are intimately linked 
within the mind of the person who possesses them. The two however differ in how easily they can be shared with others. For instance, Nonaka and Konno [15] suggest that explicit knowledge can be expressed in words and numbers and therefore shared with others in the form of specifications or formulas, whereas tacit knowledge is highly individual and hard to formalize, and therefore difficult to communicate to others. Sharing tacit knowledge is further complicated as, in addition to technical know-how and mastery of a certain task, it may also encompass mental models, values and beliefs so ingrained in us that we take them for granted [16]. On the other hand, while also recognizing the challenges associated with sharing tacit knowledge, Von Krogh et al. [17] note that tacit knowledge is "the most important source of innovation" (p. 2). In order for an individual to use the tacit knowledge they possess for the purposes of innovation, the individual is required to undergo a personal process of self-renewal through learning new information and processing it into valuable knowledge [16]. For innovation to occur, an individual involved in the innovation process needs to thus engage in behaviors that enable them to renew their thinking and knowledge they hold. Cook and Brown [18] suggest such renewal of knowledge occurs as the individual interacts with the world around them, actively using the knowledge they already hold. Grisold and Peschl [19] however note that such changing of one's knowledge is particularly challenging as we are driven by our past experiences, whereas creating new knowledge requires to some degree a departure from existing knowledge.

In sum, as our foundation, we take the view typically used in management literature that knowledge is something an individual possesses [18]. We however blend this view with a practice-oriented view of knowledge, recognizing the continuously evolving nature of knowledge. More specifically, we view knowledge as the state of mind of an individual [12], molded over time by practice and thus being "at any given time what practice has made it" [20].

\subsection{Knowledge in IT development teams}

The development of innovative digital applications within DILs normally occurs in teams which is why it is essential for us to consider existing insights of knowledge in teams. More specifically, innovation is driven by exchanging and combining knowledge [21]. In DILs such knowledge exchange and combination occur as a cross-functional team is brought together and the team members work closely together over the course of a project. The teams in DILs tend to include members with distinct specialisms ranging from developers and IT architects to designers and business specialists. Empirical studies highlight the importance of having such diverse knowledge bases within software development teams [22] when generating new ideas for software solutions [23, 24]. Walz, Elam and Curtis [23] highlight the role of knowledge particularly in the early stages of the software development process, calling it the "raw material" (p. 63) of any team tasked with software design. Both functional and technical knowledge are needed in order to build a new software application which fits the requirements of the business and works well technically.

Focusing on the knowledge that each individual team member possesses, Espinosa et al. [25] distinguish between taskwork and teamwork knowledge. Taskwork knowledge relates to having knowledge which is specific to the task at hand, whereas teamwork knowledge relates to having knowledge about other members of the team, understanding how they work and how the team can operate together as a unit [25]. Taskwork knowledge can include for instance technical knowledge of the technology platform that an application is being developed on, or functional knowledge about the business or functional area a solution is being built for. In turn, teamwork knowledge can include knowledge about the project methodology and how the team works and coordinates its work on a day-to-day basis. Put simply, taskwork knowledge is a team's raw material for idea generation and development of an innovative software application, whereas teamwork knowledge enables the team to put that raw material into use by working in a certain manner. Both are therefore essential to the software development process. Moreover, both types of knowledge exist in explicit and tacit format. Technical and functional knowledge relating to the task can to a certain extent be made explicit, for instance through formal training on the technology platform or documentation of a business process. Similarly, teamwork knowledge can be explicit in the form of methodology guidelines that spell out the team's ways of working, but it also exists in tacit form as the team members learn what their preferred ways of working together are. We expect a sufficient level of teamwork knowledge to be of particular importance in digital innovation projects where project teams are typically cross-functional teams consisting of individuals with vastly distinct specializations.

DILs can be seen to represent a particular organizational context where exchanging and combining specific pieces of knowledge can result in value-adding innovations [26]. In this way DILs can be seen as what Nonaka et al refer to as 'bas' or shared spaces dedicated to integrating specialized knowledge 
to allow for the creation of new knowledge [15]. However, developing innovative software in a DIL presents two unique somewhat paradoxical requirements for knowledge compared with the traditional software development process. Firstly, the team operates somewhat separately from the rest of the organization which may hinder its ability to share knowledge with the rest of the organization. Second, and somewhat paradoxical to the first point, the team needs to a certain degree insulate itself from organizational level knowledge which manifests itself in the form of organizational routines and processes as we describe next.

\subsection{Knowledge on organizational level}

While to a certain degree autonomous, DILs are set within the internal context of the existing organization, requiring us to examine the role of the existing organization in terms of knowledge that is used within the DIL. In his original paper describing exploration and exploitation, March [3] notes that "organizations store knowledge in their procedures, norms, rules and forms" (p. 73). Knowledge relating to this so-called organizational code accumulates over time as routines form and evolve to guide individuals' behavior in scenarios that repeat over time [27]. Put simply, the organizational code includes implicit instructions on the "way things are done around here" [27 p. 327]. This organizational code has also been associated with the wider notions of organizational culture [28] and institutional knowledge [29]. For instance, Reich's [29] description of institutional knowledge draws parallels with the organizational code described by March [3] in noting that this knowledge "is a mix of the history, the power structure, and the values of the organization" (p. 9). The underlying objective of separating DILs structurally from the rest of the organization is to enable project teams within the DIL to depart from this organizational code which could impose existing, rigid and formal organizational routines and procedures on them, and to allow them to deploy more agile ways of working and flexible processes better suited for innovation [3].

While knowledge relating to the organizational code is evidently associated with the organizational level of analysis, March [3] notes that individuals learn this organizational code through socialization as they join an organization. Socialization can be understood as an organic transfer of tacit knowledge from one individual to another [15]. Rather than through written or verbal instructions, socialization occurs organically when working together, especially when co-located in the same physical space [15] and ultimately results in similar ways of thinking and working. Individuals therefore learn and adapt to the organizational code and way of working as they work in an organization over time. Moreover, it influences how individuals view the organizational environment, search for alternatives and arrive at decisions when performing tasks within the organization [30]. Finally, this knowledge can change through individuals' experiences and acquisition of new knowledge, resulting in ultimately the organization itself learning [27].

Having reviewed the key aspects of knowledge as an individual's state of mind, its role when developing innovative software applications in teams and its relation to the internal organizational context, we now turn to delineating six types of knowledge that we argue are relevant for DILs dedicated to IT exploration. In doing so, we draw on the underlying concepts of knowledge and the approach of separating exploration and exploitation from each other as described in this and the preceding two sub-sections.

\section{Conceptualizing knowledge in DILs}

As mentioned before, our conceptualization is grounded in the point of view of an individual that is transferred into a DIL from the existing organization for the duration of a digital innovation project. This point of view is based on a recent study by Holotiuk and Beimborn [10] which examines how DILs share knowledge with the rest of the organization. The authors describe how individual employees are transferred from their base roles in the existing organization into the DIL. This transfer allows the DIL and the rest of the organization to share knowledge both during and after a project. More specifically, the individual moving between the two units plays a key role in, on the one hand, providing the DIL with relevant knowledge to use during the project and, on the other hand, taking the newly created knowledge back to the rest of the organization after the project. In terms of exploration and exploitation, the individual switches between the two activities as they move first from the existing organization (exploitation) into a digital innovation project in the DIL (exploration) and later back into their base role in the existing organization (exploitation) once the project has come to an end. Nonetheless, what remains obscure are the types of knowledge that are involved in this context of switching between exploration and exploitation, and the DIL and the existing organization. Our conceptualization sheds light on this question.

\subsection{Knowledge Types}


Our conceptualization is summarized in Table 1.

To reflect the two distinct way of working in the two separated units-the DIL and existing organizationour first knowledge category consists of ways of working knowledge. This category draws inspiration from the organizational code discussed by Levinthal and March [4] and the concept of teamwork knowledge by Espinosa et al. [25]. This category is divided into two knowledge types: exploration ways of working knowledge and exploitation ways of working knowledge. Exploration ways of working knowledge refers to new project methods and approaches that are more suited for digital innovation, such as design thinking, agile, scrum and lean start-up, which are primarily deployed in the DIL. In turn, exploitation ways of working knowledge refers to existing methods, routines and related norms that are present and used within the rest of the organization. Notably, the exploitation ways of working knowledge type is influenced heavily by the organizational code and "the way things are done around here" which an employee is over time socialized to and which guides their behavior within the organization $[3,27]$.

An individual who is transferred into the DIL for a project may be new to innovation-related methods and ways of working, needing to learn exploration ways of working knowledge as they join a DIL project team. This learning may occur through a mix of being formally taught a certain method or approach for the project and informally through working within the team. Exploration ways of working knowledge may vary across projects as one method or approach may be better suited to a specific project than another. This implies that even with some familiarity of typical approaches to innovation, an individual joining the
DIL for a specific project is likely to need to acquire some exploration related ways of working knowledge.

We propose that having appropriate exploration ways of working knowledge enables an individual to work in a DIL team and allows the team to operate together effectively as a unit during a digital innovation project $[22,31]$.

In turn, the same individual is required to intentionally forget the exploitation related ways of working knowledge they hold for the duration of the DIL project. This forgetting allows for free and creative thinking during the project. Intentionally forgetting exploitation related ways of working knowledge is particularly relevant in a digital innovation context where individuals are expected to think outside the box for new ideas, envision new organizational dimensions (e.g. new business models, products, customer segments), and consider a range of solution alternatives [32] all the while contributing to the building of a solution on a technology platform that is new to them. This intentional forgetting may be particularly challenging for individuals with a long working history in the existing organization [10]. Failing to intentionally forget this knowledge while working in the DIL may continue to direct or restrict their ways of thinking or working, hindering creativity and innovation.

Our second knowledge category consists of subject matter knowledge. This knowledge is further split into technical and business knowledge types, both of which are further split into exploitation and exploration. This results in four separate knowledge types as follows: exploitation and exploration technical knowledge, and exploitation and exploration business knowledge.

Table 1. Knowledge type and behavior conceptualization

\begin{tabular}{|c|c|c|c|c|}
\hline \multirow{2}{*}{\multicolumn{2}{|c|}{ Knowledge Category \& Type }} & \multirow[b]{2}{*}{ Description } & \multicolumn{2}{|c|}{ Knowledge behaviors } \\
\hline & & & $\begin{array}{c}\text { During DIL } \\
\text { project }\end{array}$ & $\begin{array}{l}\text { After DIL } \\
\text { project }\end{array}$ \\
\hline \multirow{2}{*}{ 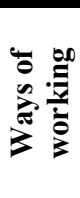 } & Exploration & $\begin{array}{l}\text { Knowledge relating to methods and ways of working } \\
\text { associated with digital innovation. }\end{array}$ & Learn, apply & $\begin{array}{c}\text { Intentionally } \\
\text { forget / } \\
\text { selectively apply }\end{array}$ \\
\hline & Exploitation & $\begin{array}{l}\text { Knowledge about methods and ways of working in the } \\
\text { existing organization. }\end{array}$ & $\begin{array}{l}\text { Intentionally } \\
\text { forget }\end{array}$ & Apply \\
\hline \multirow{4}{*}{ 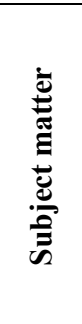 } & Exploitation technical & $\begin{array}{l}\text { Knowledge relating to legacy system landscape and } \\
\text { applications within the existing organization. }\end{array}$ & $\begin{array}{l}\text { Intentionally } \\
\text { forget }\end{array}$ & Apply \\
\hline & Exploration technical & $\begin{array}{l}\text { Knowledge relating to key technologies of digital } \\
\text { transformation. }\end{array}$ & Learn, apply & Apply \\
\hline & Exploitation business & Knowledge relating to the existing business. & Apply & Apply \\
\hline & Exploration business & $\begin{array}{l}\text { Knowledge relating to the business aspects of the } \\
\text { digital innovation developed as part of the DIL project. }\end{array}$ & Learn, apply & Apply \\
\hline
\end{tabular}


This knowledge category draws inspiration from the concept of taskwork by Espinosa et al. [25] and the more general notion of IT development projects leveraging both business and technical knowledge. We however expand this notion by further distinguishing between business and technical knowledge relating to exploration or exploitation. This distinction allows us to better examine how individuals should approach each type of knowledge. We propose that an individual possessing subject matter knowledge (in one or more domains) enables them to effectively contribute to a DIL team's tasks by providing specialized knowledge input into the DIL team's work.

Exploitation technical knowledge refers to knowledge about the legacy system landscape and applications that are used in the existing organization. In contrast, exploration technical knowledge refers to knowledge about new technologies that are used primarily to drive digital transformation, such as social, mobile, analytics, cloud and Internet of Things (SMACIT) technologies [33]. Exploitation business knowledge refers to deep business knowledge of elements such as existing business models, markets, processes, customers or products. This knowledge is typically provided by a business specialist with extensive operational experience in one specific or several closely related business areas within the existing organization. In turn, exploration business knowledge refers to new business knowledge that is created during the digital innovation project in the DIL as an idea for a new application is generated and developed into a digital solution. Depending on the project this knowledge may include elements such as new value propositions, new customer segments, new products or services or internal processes and their transformation due to a digital solution that has been developed as part of a DIL project.

While our conceptualization delineates across these six types of knowledge, we recognize that they are closely entangled. Moreover, while each team member may possess extensive knowledge of one specific type at the start of the project (e.g. a business specialist within a DIL has deep exploitation business knowledge, whereas a developer has deep exploration technical knowledge), as the team works closely together during the project, team members will acquire varying amounts of each knowledge type, with the likely exception of exploitation ways of working and exploitation technical knowledge. Drawing on the idea of knowledge being rooted in the action of an individual interacting with the world around them, [18] we expect the knowledge an individual possessed prior to a DIL project to also evolve during the project. In this way, individual knowledge belonging to each type can even be seen as something living and dynamic, rather than fully static and absolute [19].

The knowledge behaviors capture how individuals can manage the six types of knowledge. By learning we refer to the acquisition of knowledge either by formal instruction or experience. In turn, by applying knowledge we refer to actively using knowledge when pursuing a task. Finally, by intentionally forgetting we refer to actively ignoring certain type of knowledge and attempting to move away from it while pursuing a task. We should highlight that intentional forgetting does not imply fully discarding existing knowledge, but rather temporally departing from it to reduce its influence when creating new knowledge [34].

\subsection{Propositions}

Our conceptualization can be captured by six core propositions which relate the knowledge types with knowledge behaviors expected from an individual DIL member during and after a DIL project.

During a DIL project. While working on a project in the DIL, it is essential for the individual to learn the basic elements of the technology that the project is based on, gaining exploration technical knowledge. Furthermore, to be able to effectively operate in the team, the individual needs to learn exploration ways of working knowledge. Individuals temporarily transferred into the DIL from the rest of the organization are typically chosen based on their specialist knowledge in a particular business area and are thus expected to share and apply this exploitation business knowledge with the rest of the team. Finally, a transferred individual needs to intentionally forget the exploitation ways of working knowledge they possess and rather apply their newly learnt exploration ways of working knowledge allowing for creativity and experimentation of new ideas for digital innovation. In summary, our first set of propositions is as follows:

During a DIL project, an individual needs to:

$\mathrm{P} 1_{\mathrm{a}}$...learn and apply exploration technical knowledge and exploration ways of working knowledge, and; and;

$\mathrm{P} 1 \mathrm{~b}$...to apply exploitation business knowledge,

$\mathrm{P} 1_{\mathrm{c}}$...to learn exploration business knowledge, and;

$\mathrm{P} 1_{\mathrm{d}}$...to intentionally forget the exploitation ways of working knowledge and exploitation technical knowledge as they work within the DIL project team with the objective of developing an innovative digital solution.

The final proposition is of particular importance as, notably together with $\mathrm{P} 1_{\mathrm{a}}$, it allows an individual to 
gain the right mindset for digital innovation while working on a digital innovation project in the DIL.

Effectively managing the six distinct types of knowledge presents a potentially challenging task for an individual transferring between the DIL and the organization. The structural separation of the DIL aims to achieve a separation from the existing knowledge base of the organization. An employee in the organization will however have learnt and internalized the existing organizational code over time holding it as tacit exploitation ways of working knowledge which may direct their ways of doing things while performing tasks. To allow for creativity and free thinking whilst working on a project in the DIL, they are therefore required to intentionally forget this knowledge. On the other hand, the same individual is expected to actively apply their exploitation business knowledge, such as knowledge about certain business processes or products whilst working in the DIL. Furthermore, an individual in the DIL needs to be able to integrate this highly specific business knowledge with exploration technical knowledge relating to a new digital technology, while also learning and applying exploration ways of working knowledge to effectively collaborate with their team. This is all the while they are simultaneously creating and learning exploration business knowledge as they develop an innovative digital solution as part of the DIL project.

After a DIL project. Separation from the existing organizational code is required during the early stages of generating ideas for new digital solutions. However, once a successful prototype of an innovation has been developed, an individual needs to apply all types of knowledge, with the exception of exploration ways of working knowledge, to successfully integrate it into the organization. Exploitation ways of working knowledge is of particular importance to help embed the innovation into the organization as it facilitates selling the innovation in the right way [10]. Knowing the organization well will enable an individual transferring from the DIL back into the rest of the organization to effectively identify suitable organizational units or user groups for piloting and scaling the innovation. In addition, this knowledge will allow them to navigate organizational politics and power structures to gain the right kind of buy-in and support for the new innovation to be embedded in the existing organizational routines and ways of working. To achieve this and to align with the rest of the organization, the individual needs to intentionally forget the exploration ways of working knowledge they gained during the project in the DIL. On the other hand however, the individual may choose to apply some of their newly learnt exploration ways of working in their base role when appropriate, consequently promoting a culture of agility in the rest of the organization and advancing organizational learning. Such scenarios could include for instance subsequent (non-DIL) projects, with small teams where inertia and resistance towards change is likely to be lower. Our second set of propositions is as follows:

After a DIL project, an individual needs to:

$\mathrm{P2} \mathrm{a}_{\mathrm{a}}$ : ...apply all types of knowledge except exploration ways of working knowledge, and;

$\mathrm{P} 2 \mathrm{~b}$ : ...intentionally forget exploration ways of working knowledge to facilitate the embedding of new innovation into the organization, but may also selectively apply it when appropriate

....as they move back into the existing organization to facilitate the embedding of the digital innovation and continue their work in their base role.

\section{Illustrating the knowledge types}

We now illustrate our conceptualization and propositions with two vignettes. The vignettes are based on nine expert interviews that were performed in May-October 2019 as part of a wider research project. Full interview details are omitted due to space restrictions, but are available online at: https://drive.switch.ch/index.php/s/ShCTHWVosBxW DV4. All interviewees had extensive experience of interacting with and working in DILs. The interviewees described a project that had recently been carried out in the DIL of a client organization, an incumbent in the chemical manufacturing industry. The interviewees were able to describe how knowledge played a part not only in the projects' success, but also in the challenges the team encountered.

Both vignettes relate to a software application development project referred to as the DI (digital innovation) Project. The project had no clearly defined objective to begin with. It was very explorative, largely with the idea: "to develop something new and innovative" on the chosen technology platform. The project was carried out in the organization's DIL which had been set up to rapidly ideate and develop prototypes for digital software applications, thus enhancing the organization's digital innovation capability. The DIL was based in an office space dedicated and designed for innovation, physically separate from the existing organization. The DIL fell under the governance of an innovation department with permanent staff members who oversaw projects carried out in the DIL.

The DI Project team was a small cross-functional team with three developers (from the software platform vendor), a product owner, a scrum master and a project manager. The team's scope of work included the first stages of an innovation process: from generating an 
idea for a new software application to developing a first prototype of the application that could then be transferred into the rest of the organization for scaling. The DI Project was scheduled to last 3 months.

\subsection{Vignette 1: Exploitation and Exploration Ways of Working Knowledge}

The below excerpt highlights how an individual team member failed to temporarily intentionally forget exploitation ways of working knowledge and hindered the DIL team's work.

The DIL had been separated from the existing organization so that it could deploy iterative and agile ways of working suited for innovation. However,the DIL was still in its early stages and there was little exploration ways of working knowledge available within the existing organization. Consequently, the organization had hired an external contractor as the product owner for the DI Project to lead the team's efforts. The contractor had been chosen largely due to their experience on innovation at other large organizations as a result of which they had a broad view on how innovation was approached in other incumbent organizations. The contractor had not worked at the case organization before, nor in the particular industry, but they possessed extensive exploration ways of working knowledge due to their experience with innovation projects at other incumbents.

As the work started, the project manager quickly challenged the experimental ways in which the product owner encouraged the team to work. As an example, the interviewees described how the project manager insisted the team clearly define deliverables that could be listed and tracked against a purchase order. The team found this extremely challenging as the project was explorative and neither the subject area nor objective were clearly specified from the outset. Defining deliverables therefore seemed like an unnecessary administrative task. More specifically, as the project manager who had been transferred into the DIL from the existing organization failed to intentionally forget the exploitation ways of working knowledge they possessed, and imposed this knowledge on the team thus hindering the their work.

\section{Discussion}

Vignette 1 demonstrates how the project manager failed to an extent to intentionally forget their exploitation ways of working knowledge but rather imposed it on the team. As a result, the team's work was hindered and overburdened by unnecessary admin tasks. This vignette demonstrates that while the separation of a DIL from the rest of the organization should have provided the project manager the freedom to depart from existing routines and ways of working, they failed to do so, choosing to continue using existing procedures which were ill-suited for the explorative activities of the project team. As such, setting up an organizational unit dedicated to IT exploration on its own may not be sufficient to effectively enhance an organization's digital innovation capability. Rather individuals within the DIL who possess extensive exploitation ways of working knowledge need to be able to temporarily intentionally forget this knowledge while working in the DIL, and learn and apply exploration ways of working knowledge instead.

\subsection{Vignette 2: Exploitation Business Knowledge}

The following excerpt highlights the lack of exploitation business knowledge within a DIL project team during the DI Project.

Rather than a business specialist being fully allocated and transferred to the team for the DI Project, the team had been nominated business specialists that could provide the team with input and feedback on their progress when necessary. The business specialists were therefore not co-located with the project team on a daily basis, but the team could request meetings with them when needed instead.

As the team started working together, it quickly became apparent that the team did not have sufficient 'raw material' to generate ideas for software applications that could add value to the organization. As both the developers and product owner were external to the organization, they did not possess extensive exploitation business knowledge. The business specialists who the team had been nominated to gather input and feedback from were reluctant to share their knowledge as they did not see the value of digital innovation and in particular of the application the team was developing. Furthermore, the business specialists were nervous that sharing their exploitation business knowledge (which was largely tacit knowledge about the process of developing new products) would make it explicit and consequently easy to share, ultimately democratizing it. This hindered the team's work as they had no clear view of the kind of new application that could add genuine value to the business. The interviewees also noted that getting the business specialists to join the team for several days at a time was challenging, but the few times they succeeded in doing this, productivity was significantly higher. They attributed this positive impact on the business specialists having enough time to temporarily intentionally forget the exploitation ways of working 
knowledge they possessed and to get into the mindset of working more iteratively and experimentally. Ultimately however, to obtain sufficient exploitation business knowledge over the course of the project, the team had to rely on an individual from the innovation department who had been at the organization for more than 10 years. This individual had accumulated significant insight into the business and industry, together with a future vision for the organization, but yet who was highly supportive of innovation and open to exploration ways of working.

\section{Discussion}

Vignette 2 demonstrates how not having an individual possessing a significant amount of exploitation business knowledge in the team hindered progress during the development. The knowledge was held outside the team, in the rest of the organization. This resulted in the team not being able to operate as a fully self-contained unit, but having to rely on sourcing exploitation business knowledge from outside the team. This is in contrast with the scenario described by Holotiuk and Beimborn [10] where an employee from an operational role is transferred to the DIL on a fulltime basis, rather than only contributing to the team's work sporadically and at a distance. Yet, in line with the findings of Holotiuk and Beimborn, the vignette also highlights the importance of giving an individual sufficient time to intentionally forget exploitation ways of working knowledge and to learn and apply exploration ways of working knowledge. Furthermore, it highlights the intimate entanglement of both explicit and tacit knowledge within an individual's mind: an individual needing to possess exploration ways of working knowledge to effectively make their exploitation business knowledge actionable and operable [14].

\section{Conclusion}

In this paper we have conceptualized six different types of knowledge and proposed that individuals who engage in IT exploration and exploitation-by transferring between a DIL and an existing organization-can manage them by learning, applying and intentionally forgetting. More broadly, we have argued that setting up an organizational unit dedicated to IT exploration - such as a DIL - on its own may not be sufficient to ensure fruitful digital innovation. Instead, attention must be paid to individual-level knowledge types and related behaviors during and after a DIL project. In other words, we view digital innovation as being not just about "putting together diverse bits of data and information, [but rather] a highly individual process of personal and organizational self-renewal" [16 p. 10] also requiring learning, applying and intentionally forgetting of certain types of knowledge. We have anchored our conceptualization in the view of digital innovation being ultimately an individual-level process where new knowledge is created during a DIL project with the ultimate aim of building an organization's digital innovation capability.

While offering a first view of knowledge types in a DIL context, our conceptualization requires further enrichment and validation in the future. It already, however, has implications for both researchers and practitioners in how they can think of DILs and their ability to support digital innovation. First, for IS researchers, our conceptualization provides a basis for future research on digital innovation and IT exploration in a specific structural context where these activities are separated into a separate organizational unit such as a DIL. Notably, the conceptualization opens avenues for future research at individual level, and how individuals can manage switching between IT exploration and exploitation activities. Moreover, future studies may leverage our conceptualization to analyze how individual knowledge and learning in a DIL may aggregate up to the organizational level and contribute to organizational learning. For instance, as an individual moves from the DIL back into their base role and they selectively apply exploration ways of working knowledge in the existing organization, to what extent can they be successful in encouraging a culture of agility and digitalization, and ultimately facilitating a cultural shift at organizational level [35]? Second, our conceptualization is beneficial to practitioners such as executives or project managers in charge of DILs and project teams in them. Concretely, our conceptualization raises awareness of the distinct knowledge types, which is helpful when considering individual employees to be transferred from the existing organization into the DIL and further, to help the individuals manage the various knowledge types required during the project. Our conceptualization therefore helps practitioners put together and manage teams dedicated to digital innovation.

\section{References}

[1] R. Kohli, and N.P. Melville, "Digital innovation: A review and synthesis", Information Systems Journal 29(1), 2019, pp. 200-223.

[2] K. Božič, and V. Dimovski, "Business intelligence and analytics use, innovation ambidexterity, and firm performance: A dynamic capabilities perspective", Journal of Strategic Information Systems, 2019.

[3] J. March, "Exploration and Exploitation in Organizational Learning”, Organization Science 2(1), 1991, pp. 71-87. 
[4] D.A. Levinthal, and J.G. March, "The Myopia of Learning”, Strategic Management Journal 14(S2), 1993, pp. 95-112.

[5] R. Katila, and G. Ahuja, "Something Old, Something New: A Longitudinal Study of Search Behaviour and New Product Introduction", Academy of Management Journal 45(6), 2002, pp. 1183-1194.

[6] D. Leonhardt, I. Haffke, J. Kranz, and A. Benlian, "Reinventing the IT Function: The Role of IT Agility and IT Ambidexterity in Supporting Digital Business Transformation", Proceedings of the European Conference on Information Systems, (2017), 968-984.

[7] M.L. Tushman, and C.A. O'Reilly III, "Ambidextrous Organizations: Managing Evolutionary and Revolutionary Change", California Management Review 38(4), 1996, pp. 8-29.

[8] D. Lavie, U. Stettner, and M.L. Tushman, "Exploration and exploitation within and across organizations", Academy of Management Annals 4(1), 2010, pp. 109-155.

[9] C.A. O'Reilly III, and M.L. Tushman, "Organizational Ambidexterity: Past, Present, and Future", Academy of Management Perspectives 27(4), 2013, pp. 324338.

[10] F. Holotiuk, and D. Beimborn, "Temporal Ambidexterity: How Digital Innovation Labs Connect Exploration and Exploitation for Digital Innovation", Proceedings of the International Conference on Information Systems, (2019), 1-17.

[11] P. Zapadka, "Digital Innovation in Industrial-Age Firms: Managing the Balancing Act of Knowledge Integration”, ECIS 2020 Proceedings, (2020).

[12] M. Alavi, and D.E. Leidner, "Review: Knowledge Management and Knowledge Management Systems: Conceptual Foundations and Research Issues", MIS Quarterly 25(1), 2001, pp. 107-136.

[13] R.M. Grant, "Toward a Knowledge-Based Theory of The Firm", Strategic Management Journal 17(S2), 1996, pp. 109-122.

[14] J.S. Brown, and P. Duguid, "Organizing knowledge”, California Management Review 40(3), 1998, pp. 90-111.

[15] I. Nonaka, and N. Konno, "The Concept of 'Ba': Building a Foundation for Knowledge Creation", California Management Review 40, 1998, 40-54.

[16] I. Nonaka, and H. Takeuchi, The knowledgecreating company, Oxford University Press, 1995.

[17] G. Von Krogh, K. Ichijo, and I. Nonaka, "Enabling Knowledge Creation: How to Unlock the Mystery of Tacit Knowledge and Release the Power of Innovation", In Enabling Knowledge Creation. Oxford Scholarship Online, 2000.

[18] S.D.N. Cook, and J.S. Brown, "Bridging Epistemologies: The Generative Dance between Organizational Knowledge and Organizational Knowing”, Organization Science 10(4), 1999, pp. 381-400.

[19] T. Grisold, and M.F. Peschl, "Why a Systems Thinking Perspective on Cognition Matters for Innovation and Knowledge Creation. A Framework towards Leaving behind Our Projections from the Past for Creating New Futures", Systems Research and Behavioral Science 34(3),
2017 , pp. $335-353$.

[20] W.J. Orlikowski, "Knowing in practice: Enacting a collective capability in distributed organizing", Organization Science 13(3), 2002, pp. 249-273.

[21] J. Nahapiet, and S. Goshal, "Creating organizational capital through intellectual and social capital", Academy of Management Review 23(2), 1998, pp. 242-266.

[22] S. Faraj, and L. Sproull, "Coordinating Expertise in Software Development Teams", Management Science 46(12), 2000, pp. 1554-1568.

[23] D.B. Walz, J. Elam, and B. Curtis, "Inside a Software Design Team: Knowledge Acquisition, Sharing, and Integration", Communitions of the ACM 36(10), 1993, pp. 63-77.

[24] A. Tiwana, and E.R. McLean, "Expertise integration and creativity in information systems development", Journal of Management Information Systems 22(1), 2005, pp. 13-43.

[25] J.A. Espinosa, S.A. Slaughter, R.E. Kraut, and J.D. Herbsleb, "Team knowledge and coordination in geographically distributed software development", Journal of Management Information Systems 24(1), 2007, pp. 135-169.

[26] A. Hund, F. Holotiuk, H.-T. Wagner, and D. Beimborn, "Knowledge Management in the Digital Area: How Digital Innovation Labs Facilitate Knowledge Recombination", Proceedings of the European Conference on Information Systems, 2019, pp. 0-15.

[27] B. Levitt, and J.G. March, "Organizational learning", Annual review of sociology 14(1), 1988, pp. 319340 .

[28] D. Laubengaier, G. Hahn, and H.-T. Wagner, "Organizational Culture and Knowledge Exchange and Combination: A Systematic Literature Review", Proceedings of the 52nd Hawaii International Conference on System Sciences 6, 2019, pp. 5498-5507.

[29] B.H. Reich, "Managing Knowledge and Learning in IT Projects: A Conceptual Framework and Guidelines for Practice", Project Management Journal 38(2), 2007, pp. 517.

[30] R.M. Cyert, and J.G. March, “A behavioral theory of the firm”, Englewood Cliffs, NJ 2(4), 1963, pp. 169-187.

[31] L. Argote, and E. Miron-Spektor, "Organizational learning: From experience to knowledge", Organization Science 22(5), 2011, pp. 1123-1137.

[32] K. Werder, and C.S. Heckmann, "Ambidexterity in Information Systems Research: Overview of Conceptualizations, Antecedents, and Outcomes", Journal of Information Technology Theory and Application 20(1), 2019, pp. $28-52$.

[33] G. Vial, "Understanding digital transformation: A review and a research agenda", Journal of Strategic Information Systems 28(2), 2019, pp. 118-144.

[34] T. Grisold, A. Kaiser, and J. Hafner, "Unlearning before Creating new Knowledge: A Cognitive Process.", Proceedings of the 50th Hawaii International Conference on System Sciences (2017), 2017, pp. 4614-4623.

[35] S. Duerr, F. Holotiuk, H.-T. Wagner, D. Beimborn, and T. Weitzel, "What Is Digital Organizational Culture? Insights From Exploratory Case Studies", Proceedings of the 51st Hawaii International Conference on System Sciences, (2018), 5126-5135. 